\title{
Hydrogen Storage in Chemically Reducible Mesoporous and Microporous Ti Oxides
}

\section{(Supporting Information)}

\author{
Xin $\mathrm{Hu}^{\dagger}$, Boris O. Skadtchenko ${ }^{\dagger}$, Michel Trudeau ${ }^{\ddagger}$ and David M. Antonelli ${ }^{\dagger} *$ \\ ${ }^{\dagger}$ Department of Chemistry and Biochemistry, University of Windsor, 401 Sunset \\ Avenue, Windsor, Ontario N9B 3P4, Canada, and ${ }^{\ddagger}$ Chemistry and Materials, Hydro- \\ Québec Research Institute, 1800 Boul. Lionel-Boulet, Varennes, Quebec, J3X 1S1, \\ Canada
}

\section{Materials and Methods}

All chemicals, unless otherwise stated, were obtained from Aldrich. Bis (toluene) Titanium was synthesized by F. G. N. Cloke at the University of Sussex by metal vapor synthesis.

\section{Synthesis of meso- and microporous titanium oxide materials}

In a typical preparation, Titanium (IV) isoproxide (15 g, $52.77 \mathrm{mmol}$ ) was warmed with hexylamine (2.67 g, $26.39 \mathrm{mmol}$ ) until a homogeneous colorless solution was obtained. To this solution was added $75 \mathrm{~mL}$ water with stirring. Precipitation occurred immediately. $37 \% \mathrm{HCl}(0.2603 \mathrm{~g}, 2.639 \mathrm{mmol})$ was then added to the mixture. The mixture was allowed to sit at room temperature overnight without agitation before being transferred to an oven for aging at $40{ }^{\circ} \mathrm{C}$ for 2 days, $60{ }^{\circ} \mathrm{C}$ for 2 days, and $80^{\circ} \mathrm{C}$ for 4 days. The mixture then was filtered by suction filtration, and the white solid was placed into a sealed tube and put into oven at $100{ }^{\circ} \mathrm{C}$ for 2 days, $120^{\circ} \mathrm{C}$ for 2 days, and $140{ }^{\circ} \mathrm{C}$ for 2 days. The product was collected by suction filtration and washed once with a mixture of methanol (375 ml) and diethyl ether (125 $\mathrm{ml}$ ), and four times with $500 \mathrm{ml}$ methanol. The as-synthesized samples were dried at $150{ }^{\circ} \mathrm{C}$ overnight under vacuum and then treated with trimethylsilyl chloride in diethyl ether at room temperature for $4 \mathrm{~h}$. After filtration and washing with diethyl ether, the materials were dried in an oven at $150{ }^{\circ} \mathrm{C}$ overnight. 


\section{Synthesis of Li and Na reduced microporous titanium oxide materials}

To a suspension of the alkali metal (caution) in tetrahydrofuran (THF) in an inert atmosphere glovebox was added 1.0 equiv of naphthalene. The mixture was stirred until the solid was completely consumed. On the basis of 38\% $\mathrm{Ti}$ as determined from previous elemental analysis data (ref. 19), 1.0 equiv of the trimethylsilated microporous titanium was added to the THF solution. After additional stirring overnight, the reduced material was collected by suction filtration and washed several times with THF. The materials were then dried in vacuo at $10^{-3}$ Torr on a Schlenk line until all condensable volatiles had been removed.

\section{Synthesis of Bis(Toluene) Titanium reduced microporous titanium oxide materials}

Excess bis(toluene) titanium, as calculated on the basis of 38\% $\mathrm{Ti}$ in the porous oxide (ref. 19), was added to a suspension of trimethylsilated microporous titanium oxide in dry toluene under nitrogen. After 1 day of stirring to ensure complete absorption of the organometallic, the reduced material was collected by suction filtration under nitrogen and washed several times with toluene. The resulting blue black material was dried in vacuo at $10^{-3}$ Torr on a Schlenk line until all condensable volatiles had been removed.

\section{$\mathrm{H}_{2}$ sorption measurements}

The hydrogen adsorption/desorption isotherms have been measured by a computer controlled commercial 'Gas Reaction Controller’ manufactured by the Advanced Materials Corporation of Pittsburgh, PA. Highly purified hydrogen (99.9995\% purity) was used as the adsorbent. Typically the mass needed for the hydrogen sorption measurement is $500-1000 \mathrm{mg}$. The size of the sample chamber is 2.5 cc. Lightly packed powder materials were used for all measurements. Before all measurements the materials were degassed at $200^{\circ} \mathrm{C}$ under high vacuum for at least one day in order to remove any physisorbed water or volatile impurities. A simplified sketch of this apparatus is shown in Figure S1. The temperature of the gas reservoir is measured by two AD590 IC thermometers that are calibrated against a standard mercury 
thermometer within $0.1 \mathrm{C}$ at room temperature. The sample temperature is measured with type $\mathrm{K}$ thermocouple by converting voltage reading to temperature according to ITS-90 (The International Temperature Scale of 1990). The limits of error are $2{ }^{\circ} \mathrm{C}$ or $0.75 \%$ above $0{ }^{\circ} \mathrm{C}$ and $2{ }^{\circ} \mathrm{C}$ or $2 \%$ below $0{ }^{\circ} \mathrm{C}$. The pressure of both gas reservoir and sample chamber is measured by Heise model HP0 pressure transducer, which has a full scale range of 1500 psi (about $100 \mathrm{~atm}$ ). The accuracy of this transducer is rated to be $0.05 \%$ of the full scale including non-linearity, drift, and hysteresis. The GRC operates by admitting an appropriate amount of gas to the reservoir and determines its molar amount from its pressure and temperature. The system then manipulates the valves between the reservoir and the reaction chamber and transfers a desired amount of the gas from the reservoir to the gas reaction chamber. After equilibrium is attained, the system re-calculates the number of hydrogen molecules. The number missing from the gas phase corresponds to the number of molecules absorbed by the sample. The system employs a modified Benedict-Webb-Rubin equation of state in calculating the amount of absorbed hydrogen from the pressure, temperature, and volume. The apparatus gradually increases the hydrogen pressure to the maximum specified value, while summing the sorbed hydrogen. The amount of hydrogen released from the sample is then determined by pumping out the gas reservoir and gradually bleeding hydrogen from the sample chamber into the gas reservoir. During the test process the sample chamber was immersed in liquid nitrogen, and the liquid level was maintained unchanged.

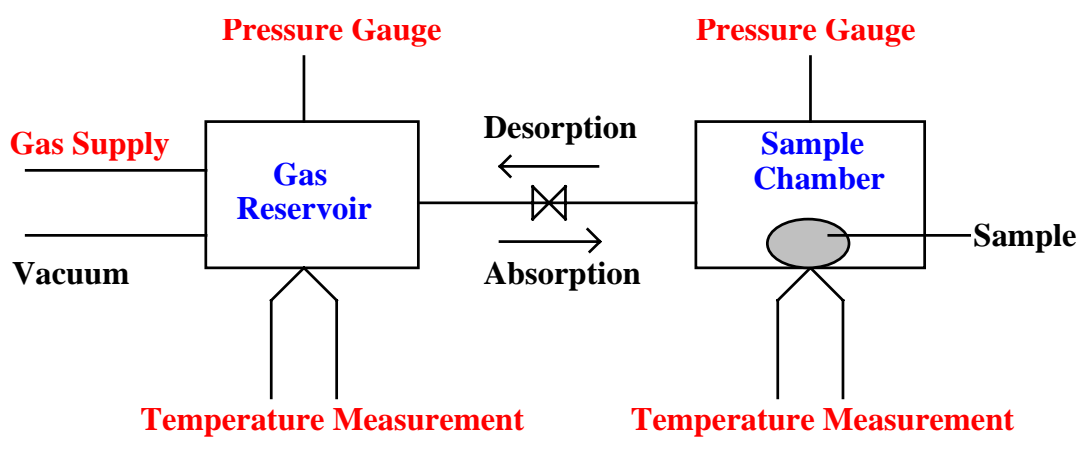

Figure S1. Schematic drawing of the GRC system.

This system effectively measures the pressure of the hydrogen in the sample chamber and then calculates the number of moles on the basis of this pressure, the temperature 
and the volume, which is arrived at by subtracting the sample volume (obtained by direct input of the sample weight and density) from the empty chamber volume. If the density used is the skeletal density measured by a pycnometer, the compressed hydrogen within the pores is treated as part of the sample chamber volume, but if the apparent density of the sample is used instead, the compressed gas in the pores is treated as having been absorbed by the sample along with the hydrogen physisorbed to the walls of the skeletal structure. The advantage of this system is that you do not have to make assumptions on the void space volume of the material to obtain the total amount of $\mathrm{H}_{2}$ in the system including the compressed gas in the void space, which is crucial in determining the amount of useful fuel stored in the material. Most other Sieverts apparatuses work by automatically subtracting out the compressed gas in the pores, obviating the use of the pycnometer, but making determination of the compressed gas in the pores more ambiguous. Gravimetric adsorption (equivalent to what is often called "excess storage”) and total storage are measured directly, while volumetric densities for both values are calculated using the apparent densities.

\section{Characterization}

XRD patterns $(\mathrm{Cu} \mathrm{K} \alpha)$ were recorded on a Siemens D5000-20 diffractometer.

Nitrogen adsorption and desorption data were collected on a Micromeritics ASAP 2010. TEM images were obtained by using a H9000 HR-TEM operated at $300 \mathrm{kV}$. The skeletal densities of the samples were measured by a Quantachrome Micro-Ultrapycnometer 1000 using helium gas. Determination of apparent density of the material is a common practice of the laboratory, simply divided the mass of the packed powders by the volumes they occupied.

Enthalpies of adsorption were calculated using a variant of the Clausius-Clapeyron equation taking both the $77 \mathrm{k}$ and $87 \mathrm{k}$ hydrogen adsorption data.

$\ln \left(\frac{P_{1}}{P_{2}}\right)=\Delta H_{a d s} * \frac{T_{2}-T_{1}}{R * T_{1} * T_{2}}$

Where $\quad P_{n}$ : Pressure for isotherm $n$

$T_{n}$ : Temperature for the isotherm $n$

R: Gas constant 
Pressure as the function of the amount adsorbed was determined by using exponential fit for each isotherm, the first ten points of the isotherms were picked up and fit to the exponential equation. This exponential equation gives an accurate fit over the pressure up to $10 \mathrm{~atm}$ and with the goodness of fit $\left(\mathrm{R}^{2}\right)$ above 0.99 . The corresponding $\mathrm{P}_{1}$ and $\mathrm{P}_{2}$ at a certain amount $\mathrm{H}_{2}$ adsorbed of both temperatures can be obtained by the simulated exponential equation. Then input these numbers into the equation 1 , the enthalpies of the adsorption were calculated. 


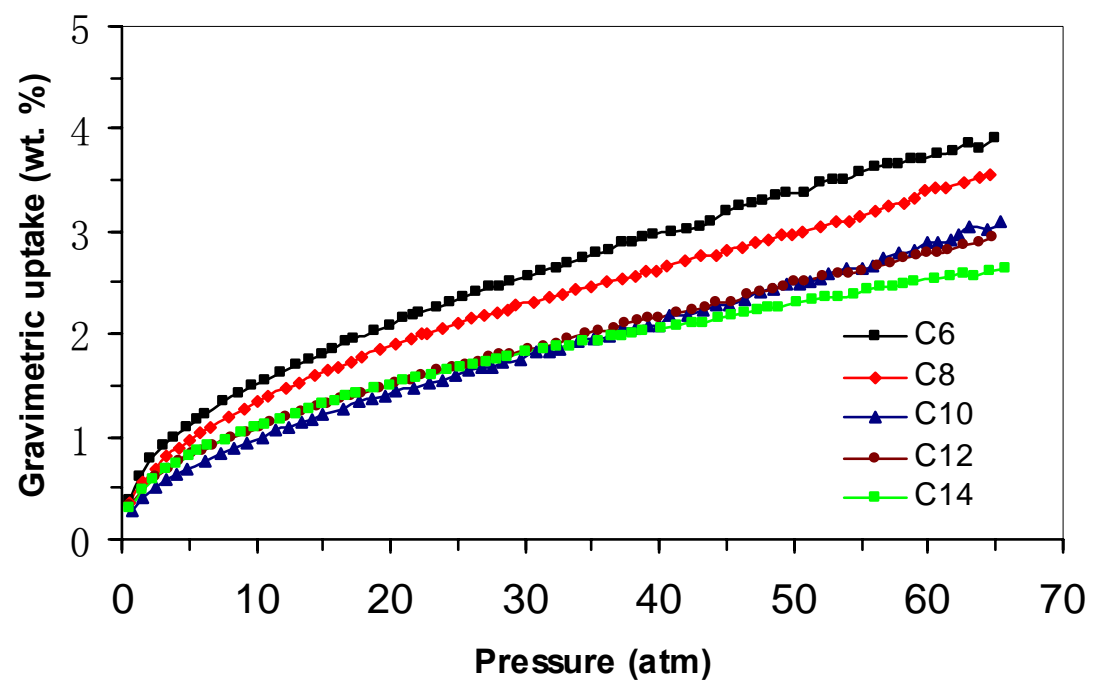

Figure S2. Hydrogen storage isotherms at $77 \mathrm{~K}$ for micro- and mesoporous titanium oxides synthesized using various templating agents in gravimetric uptake. 


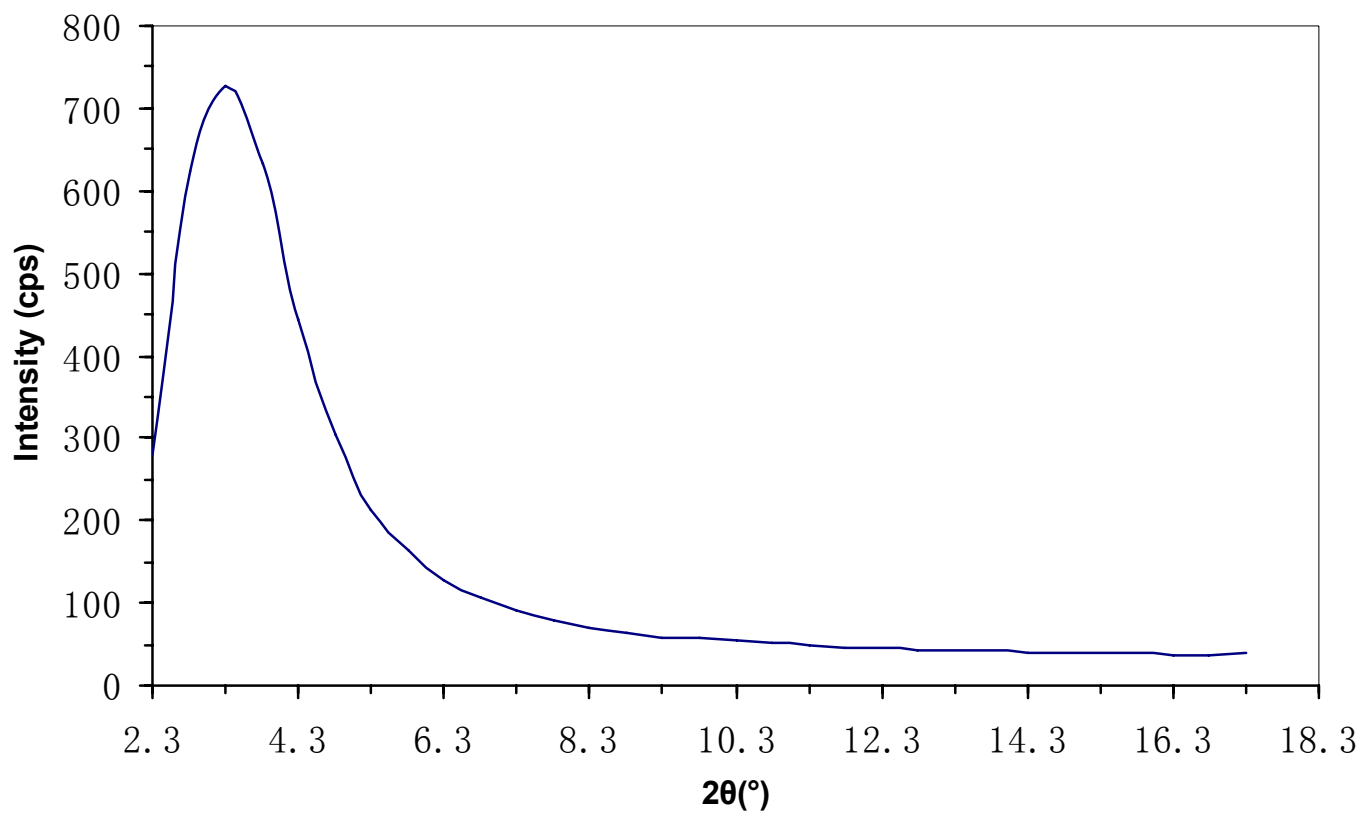

Figure S3. X-ray diffraction pattern of microporous Ti oxide synthesized with hexylamine at a 2:1 metal:surfactant ratio. 


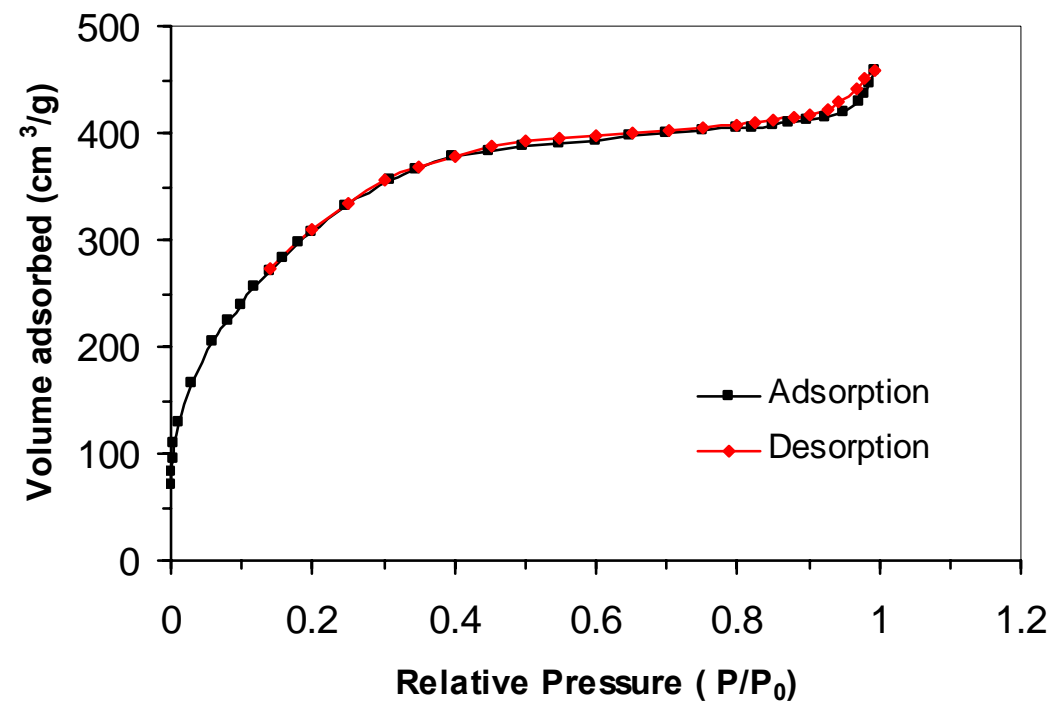

Figure S4. Nitrogen adsorption/desorption isotherm of microporous Ti oxide synthesized with hexylamine at a 2:1 metal:surfactant ratio. 


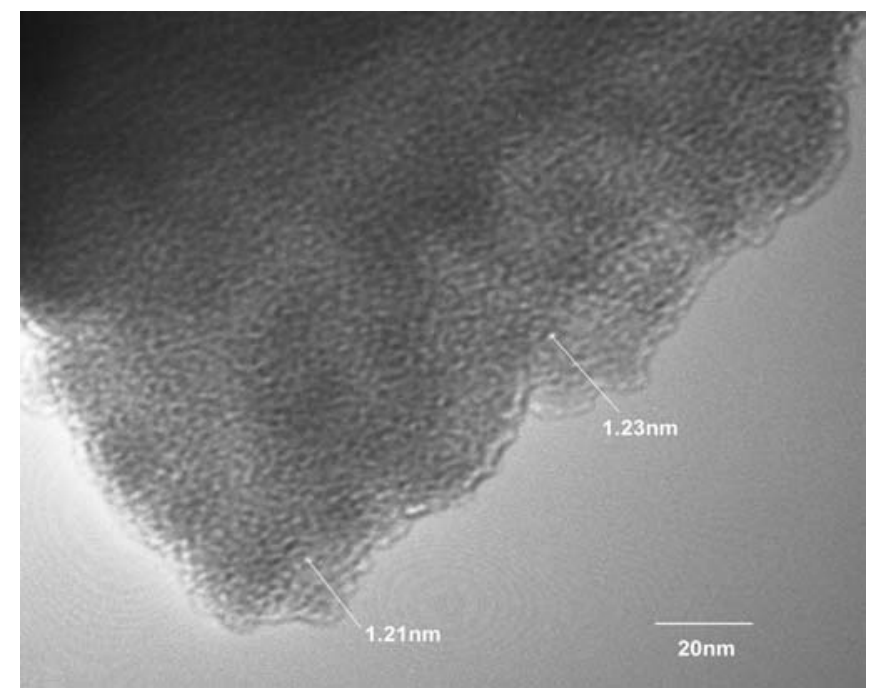

Figure S5. TEM image of microporous Ti oxide synthesized with a hexylamine template. 


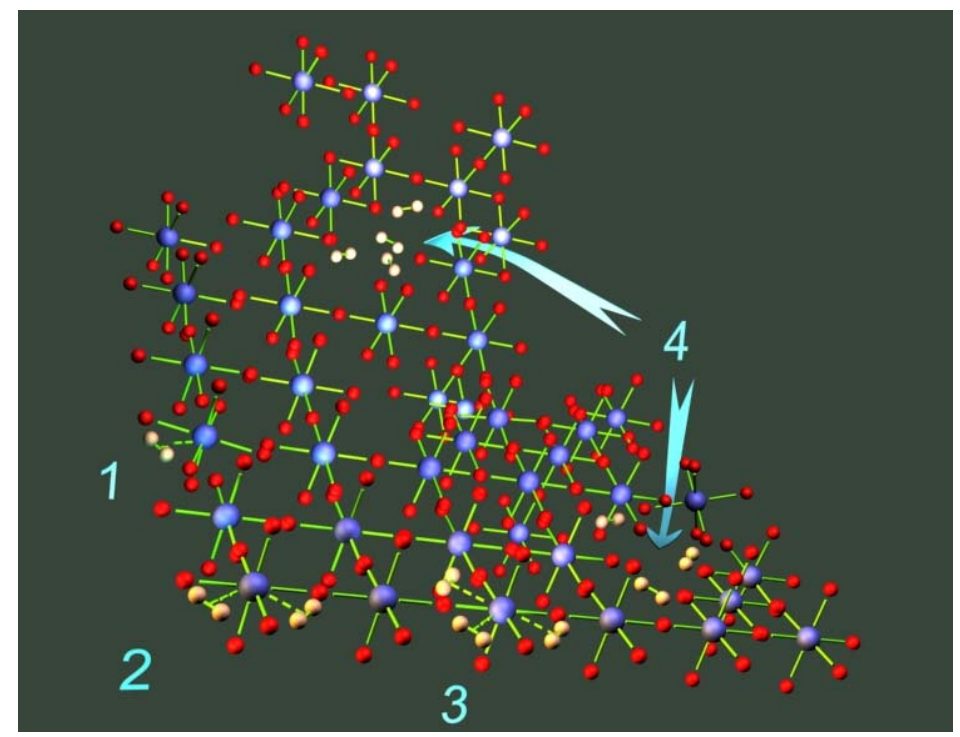

Figure S6. Schematic representation of $\mathrm{H}_{2}$ binding sites in the monolayer wall of micro-and mesoporous titanium materials, where 1, 2, and 3 refer to mono, bis, and tris dihydrogen complexes, respectively, and 4 represents the compressed gas phase in the porous voids in the solid. Reduction of the 12-electron $\mathrm{MX}_{4} \mathrm{~L}_{2}$ Ti octahedra leads to population of the $\mathrm{t}_{2 \mathrm{~g}}$ set and more effective $\pi$ back bonding to the $\mathrm{H}_{2}$ molecules. 


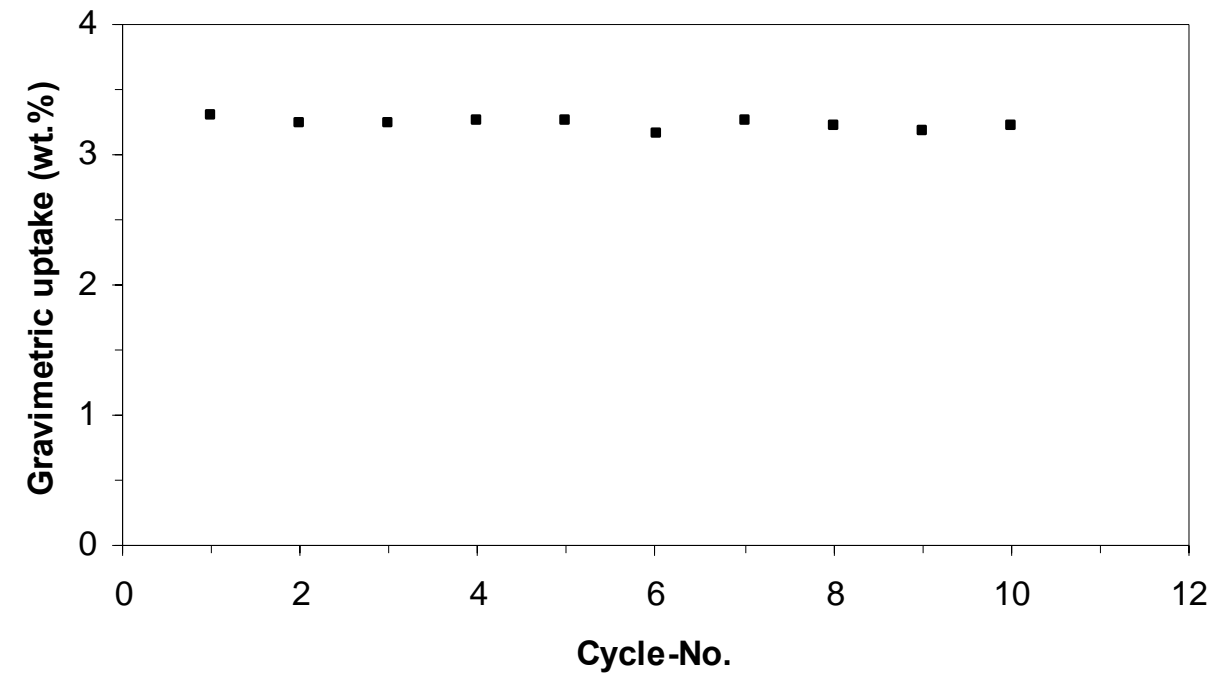

Figure S7. Hydrogen storage capacity in a 10 cycle test of the bis(toluene) titanium reduced microporous titanium oxide material at $77 \mathrm{k}$ and $65 \mathrm{~atm}$. 
Table S1. XPS results for pristine and reduced amine-templated materials.

\begin{tabular}{|c|c|}
\hline Materials & $\begin{array}{c}\text { Binding Energy of Ti } \\
\text { 3p } 1 / 2(\mathrm{eV})\end{array}$ \\
\hline Unreduced $\mathrm{Ti}$ & $37.9^{a}$ \\
\hline Li reduced $\mathrm{Ti}$ & $36.8^{a}$ \\
\hline Na reduced $\mathrm{Ti}$ & $36.4^{b}$ \\
\hline BTTi reduced $\mathrm{Ti}$ & $35.1^{a}$ \\
\hline
\end{tabular}

${ }^{a}$ From ref.19. ${ }^{b}$ From ref. 20. 
Table S2. Hydrogen sorption capacity of micro- and mesoporous Ti oxides at 77K with different surface areas synthesized using amine templating agents of various chain lengths in both gravimetric and volumetric uptake.

\begin{tabular}{|c|c|c|c|c|c|c|c|c|}
\hline Sample name & $\begin{array}{c}\text { Surface } \\
\text { area } \\
\left(\mathrm{m}^{2} / \mathrm{g}\right)\end{array}$ & $\begin{array}{c}\text { Pore size } \\
(\AA)\end{array}$ & $\begin{array}{c}\text { Apparent } \\
\text { Density } \\
(\mathrm{g} / \mathrm{ml})\end{array}$ & $\begin{array}{c}\text { True } \\
\text { Density } \\
(\mathrm{g} / \mathrm{ml})\end{array}$ & $\begin{array}{c}\text { Gravimetric } \\
\text { Absorption } \\
(\mathrm{wt} \%)\end{array}$ & $\begin{array}{c}\text { Gravimetric } \\
\mathrm{H}_{2} \text { storage at } \\
100 \mathrm{~atm} \\
(\mathrm{wt} . \%)^{b}\end{array}$ & $\begin{array}{c}\text { Volumetric } \\
\text { Absorption } \\
\left(\mathrm{kg} / \mathrm{m}^{3}\right)\end{array}$ & $\begin{array}{c}\text { Volumetric } \\
\mathrm{H}_{2} \text { storage at } \\
100 \mathrm{~atm} \\
\left(\mathrm{~kg} / \mathrm{m}^{3}\right)^{b}\end{array}$ \\
\hline C6 micro Ti & 942 & $12^{a}$ & 0.548 & 2.698 & 1.08 & 5.36 & 5.918 & 29.37 \\
\hline C8 micro Ti & 1063 & $18^{a}$ & 0.593 & 2.730 & 0.91 & 4.82 & 5.396 & 28.58 \\
\hline C10 meso Ti & 706 & 20 & 0.645 & 2.531 & 0.89 & 4.30 & 5.741 & 27.74 \\
\hline C12 meso Ti & 737 & 22 & 0.656 & 2.708 & 0.85 & 4.07 & 5.576 & 26.70 \\
\hline C14 meso Ti & 643 & 26 & 0.718 & 2.815 & 0.79 & 3.51 & 5.672 & 25.20 \\
\hline Li C6 Ti & 499 & $12^{a}$ & 0.556 & 2.508 & 0.71 & 5.63 & 3.948 & 31.30 \\
\hline Na C6 Ti & 480 & $12^{a}$ & 0.566 & 2.741 & 0.92 & 5.58 & 5.198 & 31.58 \\
\hline BTTi C6 Ti & 208 & $12^{a}$ & 0.819 & 2.835 & 1.14 & 4.94 & 9.337 & 40.46 \\
\hline
\end{tabular}

${ }^{a}$ Estimated from TEM and XRD data and previous work from (13)

${ }^{b}$ Hydrogen measurement is at the temperature of $77 \mathrm{~K}$ and $65 \mathrm{~atm}$ extrapolated to $100 \mathrm{~atm}$ with goodness of fit $\left(\mathrm{R}^{2}\right)=0.9963 \sim 0.9995$. 